\title{
A implantação de um protocolo de sepse no atendimento do pronto socorro e o impacto nos custos em um hospital privado na Região Amazônica
}

The implementation of a sepsis protocol in the emergency room and the impact on costs in a private hospital in the Amazon Region

La implementación de un protocolo de sepsis en la sala de emergencias y el impacto en los costos en un hospital privado de la Región Amazónica

Adriana de Oliveira Lameira Veríssimo ORCID: https://orcid.org/0000-0002-3497-7106 Hospital Adventista de Belém, Brasil E-mail: adylameira@gmail.com

Galvane Teixeira Gomes ORCID: https://orcid.org/0000-0003-4642-6421 Hospital Adventista de Belém, Brasil E-mail: galvanegomes@yahoo.com.br

Alzira Carvalho Paula de Souza ORCID: https://orcid.org/0000-0002-2433-0966 Hospital Adventista de Belém, Brasil E-mail: paula.alzira@yahoo.com.br

Caroline Dias Pastana

ORCID: https://orcid.org/0000-0002-5355-5515 Hospital Estadual de Santana, Brasil E-mail: pastana_carol@yahoo.com.br

Juliana Maroja Serafico Madeira ORCID: https://orcid.org/0000-0002-5317-0797 Hospital Saúde da Mulher, Brasil E-mail: juserafico@hotmail.com João Victor Moura Garcia

ORCID: https://orcid.org/0000-0001-6305-6042 Universidade Federal do Pará, Brasil E-mail: joaovgarcia98@gmail.com

Edgar de Brito Sobrinho

ORCID: https://orcid.org/0000-0001-8812-5526 Hospital Adventista de Belém, Brasil

E-mail: edgardebritosobrinho@hotmail.com

\begin{abstract}
Resumo
Objetivo: Analisar o impacto econômico da implantação de um protocolo de detecção precoce de sepse no pronto socorro de um hospital privado na cidade de Belém, Pará, Brasil Métodos: Estudo experimental, transversal, retrospectivo, realizado de março de 2015 a dezembro de 2019 de um centro de referência de alta complexidade. A implantação do protocolo ocorreu no mês de março do ano 2015. Resultados: Foram estudados um total de 257 pacientes, sendo 128 do grupo antes do protocolo (grupo AP) e 129 pertencentes ao grupo depois do protocolo (grupo DP), com proporçães semelhantes em relação ao sexo. Em relação à média de idade e desvio padrão, no grupo AP foi de $73.5 \pm 23.3$ e no grupo DP $76.0 \pm 17.0$. Após a implantação do protocolo houve redução do tempo de disfunção orgânica, taxa de mortalidade, permanência na UTI e permanência no hospital, ambas com diferença estatística de $\mathrm{p}=0,0015, \mathrm{p}=0,04, \mathrm{p}=0,012$ e $\mathrm{p}=0,0401$, respectivamente. Na avaliação dos custos hospitalares, foi identificada redução na média gasta com cada paciente tanto no custo total da hospitalização quanto no custo total referente apenas ao tempo de permanência na UTI. Conclusão: É perceptível que os custos com sepse configuram um problema de saúde pública tanto no âmbito nacional, quanto internacional. Contudo tais gastos podem ser reduzidos com a implantação de protocolos que visem padronizar a assistência prestada aos pacientes e com isso influenciar positivamente na redução do tempo de internação, custos com a internação e mortalidade.
\end{abstract}

Palavras-chave: Sepse; Protocolos; Custos de cuidados de saúde; Análise custo-benefício; Segurança do paciente.

\section{Abstract}

Objective: To analyze the economic impact of implementing an early sepsis detection protocol in the emergency department of a private hospital in the city of Belém, Pará, Brazil. Methods: Experimental, cross-sectional, 
retrospective study, carried out from March 2015 to December 2019 from a highly complex referral Center. The implementation of the protocol took place in March 2015. Results: A total of 257 patients were studied, 128 from the group before the protocol (AP group) and 129 belonging to the group after the protocol (DP group), with similar proportions. in relation to sex. Regarding the mean age and standard deviation, in the AP group it was $73.5 \pm 23.3$ and in the DP group $76.0 \pm 17.0$. After the implantation of the protocol, there was a reduction in the time of organ dysfunction, mortality rate, ICU stay and hospital stay, both with statistical difference of $p=0.0015, p=0.04, p=$ 0.012 and $p=0.0401$, respectively. In the evaluation of hospital costs, a reduction in the average spent with each patient was identified both in the total cost of hospitalization and in the total cost referring only to the length of stay in the ICU. Conclusion: It is noticeable that sepsis costs are a public health problem both nationally and internationally. However, such expenses can be reduced with the implementation of protocols that aim to standardize the care provided to patients and thereby positively influence the reduction of hospital stay, hospitalization costs and mortality. Keywords: Sepsis; Protocols; Health care costs; Cost-benefit analysis; Patient safety.

\section{Resumen}

Objetivo: Analizar el impacto económico de la implementación de un protocolo de detección temprana de sepsis en el servicio de urgencias de un hospital privado de la ciudad de Belém, Pará, Brasil. Métodos: Estudio experimental, transversal, retrospectivo, realizado de marzo de 2015 a diciembre de 2019 de un centro de derivación de alta complejidad .La implementación del protocolo tuvo lugar en marzo de 2015. Resultados: Se estudiaron un total de 257 pacientes, 128 del grupo antes del protocolo (grupo AP) y 129 del grupo posterior al protocolo (grupo DP), con proporciones similares. en relación al sexo. En cuanto a la edad media y la desviación estándar, en el grupo AP fue de $73,5 \pm 23,3$ y en el grupo DP de 76,0 $\pm 17,0$. Tras la implantación del protocolo se redujo el tiempo de disfunción orgánica, tasa de mortalidad, estancia en UCI y estancia hospitalaria, ambas con diferencia estadística de $\mathrm{p}=0,0015, \mathrm{p}$ $=0,04, \mathrm{p}=0,012 \mathrm{yp}=0,0401$, respectivamente. En la evaluación de los costes hospitalarios se identificó una reducción del gasto medio con cada paciente tanto en el coste total de la hospitalización como en el coste total refiriéndose únicamente al tiempo de estancia en UCI. Conclusión: Es notorio que los costos de la sepsis son un problema de salud pública tanto a nivel nacional como internacional. Sin embargo, dichos gastos pueden reducirse con la implementación de protocolos que tengan como objetivo estandarizar la atención brindada a los pacientes y así influir positivamente en la reducción de la estancia hospitalaria, los costos de hospitalización y la mortalidad.

Palabras clave: Sepsis; Protocolos; Costos de la atención em salud; Análisis costo-beneficio; Seguridad del paciente.

\section{Introdução}

Segundo o Instituto Latino-Americano para Estudos da Sepse (ILAES) (2015), a sepse pode ser definida como a resposta sistêmica a uma doença infecciosa, seja ela causada por bactérias, vírus, fungos ou protozoários.

No Brasil os custos da assistência ao cliente com sepse representam em torno de 9,6 mil dólares por paciente. Quando se compara as regiões brasileiras, segundo um estudo realizado por Silva et al. (2019), a região Norte é a que menos gasta com sepse no Brasil, algo em torno de R \$292,54 reais por dia com pacientes com sepse. Por outro lado, a região vizinha, Nordeste, mostrou-se a região que mais gasta diariamente com sepse, $\mathrm{R} \$ 320,70$ reais/paciente/dia (Silva et al., 2019).

O cálculo do valor gasto no manejo da sepse leva em consideração a terapêutica, diagnóstico e recursos humanos e físicos aplicados a esse paciente conforme o protocolo adotado pela instituição. Esses protocolos são criteriosos e contam com exames, medicamentos e condutas profissionais. Entretanto vale ressaltar a dificuldade em calcular o custo de condutas da equipe de enfermagem, médica e multiprofissional (Silva et al., 2019).

Em um estudo realizado em dois hospitais em Joinville/SC foi evidenciada a associação entre custo elevado e paciente mais grave, visto que, os pacientes mais graves e com desfecho em óbito correspondiam a um custo mais elevado quando comparados aos pacientes sobreviventes à sepse. Esse fato pode estar associado a aplicação de mais esforços e maior tempo de internação aos pacientes sépticos graves (Koenig et al., 2010).

As evidências demonstram que a efetiva implantação dos protocolos tem impacto na evolução dos pacientes (Ilas Online, 2015). Assim, a instauração de protocolos nos serviços de atenção é uma excelente tática para lidar com a sepse, já que tais protocolos visam abordar em um intervalo reduzido de tempo, de maneira mais eficaz e segundo a necessidade de cada paciente (Pérez et al., 2012). Estudos revelam que a implantação precoce das medidas de combate à sepse garante intervenções 
em cada fase, permitindo a aplicação de estratégias para melhorar a terapêutica (Espichán, 2010; Rocha et al., 2015; Bruhn, Pairumani \& Hernández, 2011; Romero \& Hernández, 2013; Hernández, Bruhn, Castro \& Reguera, 2012).

Diante do exposto, este estudo teve como objetivo analisar o impacto econômico da implantação de um protocolo de detecção precoce de sepse no pronto socorro de um hospital privado na cidade de Belém, Pará, Brasil.

\section{Metodologia}

Trata-se de um estudo experimental, transversal, retrospectivo, realizado de março de 2015 a dezembro de 2019, sendo braço do projeto guarda-chuva "Sepse: implicações, prevenção, custo-efetividade e a qualidade de vida dos sobreviventes" de um centro de referência de alta complexidade, de um hospital evangélico da cidade de Belém-Pará. O presente estudo possui abordagem quantitativa, a qual de acordo com Pereira, Shitsuka, Parreira e Shitsuka (2018), ocorre mediante a coleta de dados quantitativos ou numéricos por meio de medições de grandezas, e que em seguida são analisados por técnicas matemáticas como porcentagens, estatísticas, probabilidades além de métodos analíticos e numéricos.

A implantação do protocolo ocorreu no mês de março do ano 2015. Durante esse processo foram desenvolvidos guias de referência, confeccionados folhetos explicativos e fluxogramas com algoritmos, distribuídos por todos os profissionais da saúde do hospital, principalmente no setor de emergências, os mesmos estavam baseados nas diretrizes internacionais de sepse de 2012 e do Programa Brasileiro de Segurança do Paciente (Dellinger et al., 2013; Ilas Online, 2015; Brasil, 2014).

Os materiais produzidos continham informações sobre a identificação dos primeiros sinais e sintomas da sepse, a importância da abertura do protocolo de sepse e o impacto que essa ação tem sobre a mortalidade dos pacientes acometidos.

Para realizar o estudo, foram incluídos um total de 258 pacientes, escolhidos aleatoriamente em cada período estudado, com 128 no grupo AP (Antes do Protocolo) e 129 no grupo DP (Depois do Protocolo), e que apresentavam os dados quanto aos custos de internação completos. Foram selecionados adultos, maiores de 18 anos, diagnosticados com sepse, sepse grave ou choque séptico, admitidos desde o setor de emergência, ou os que foram transferidos para a UTI dentro das primeiras 24 horas do diagnóstico. Os critérios de definição de sepse foram baseados nos critérios da CSS (Quadro 1).

Quadro 1: Pacotes de terapia da Campanha Sobrevivendo a Sepse.

\section{Pacotes de 3 horas}

1 Medir o nível de lactato.

2 Obter hemoculturas antes da administração de antibióticos.

3 Inicio de antibióticos, de amplo espectro, por via intravenosa, na primeira hora do diagnóstico.

4 Administrar $30 \mathrm{~mL} / \mathrm{kg}$ de cristaloides ou o equivalente de coloides para hipotensão ou lactato $\geq 4$.

\section{Pacotes de 6 horas}

\section{(Pacientes com hiperlactatemia ou hipotensão persistente)}

1 Aplicar vasopresores (para hipotensão que não responde a ressuscitação de líquido inicial para manter una PAM $>$ $65 \mathrm{mmHg})$;

2 Em caso de hipotensão arterial persistente a pesar da ressuscitação de volume ou lactato inicial de 4mmol/L (36mg/dL):

- Medir pressão venosa central (PVC);

- Medir a saturação de oxigeno venoso central (ScvO2);

- Medir novamente o lactato.

Fonte: Campanha de sobrevivência à sepse: Diretrizes internacionais para tratamento de sepse grave e choque séptico, 2012 (Dellinger et al., 2013). 
Foram excluídos os pacientes obstétricos, pacientes com sepse encaminhados de outras instituições e quanto às disfunções orgânicas, não se considerou às secundárias a uma doença crônica.

A compilação dos dados clínicos e demográficos foi feito através das histórias clínicas dos pacientes, mediante o formulário desenhado pelo ILAS (Ilas Online, 2015) e adaptado pela gestão de qualidade do hospital em conjunto com a comissão de controle de infecção hospitalar (CCIH).

A partir do momento que o paciente com suspeita de sepse era admitido no hospital, a equipe da CCIH era acionada e eram coletados os dados no formulário pré-desenhado para esse fim. A avaliação era feita baseada no cumprimento do pacote de reanimação da CSS atualizada em 2012. O mesmo consta com requisitos que devem cumprir-se dentro das primeiras 3 e 6 horas, e que constam de intervenções diagnosticas e terapêuticas. As medidas foram consideradas aderentes ao protocolo, quando se cumpre com cada item dos pacotes, demonstrados anteriormente no Quadro 1.

As análises estatísticas foram realizadas utilizando o programa BioEstat versão 5.3. As características sociodemográficas, epidemiológicas e clínicas foram descritas usando a estatística descritiva (as variáveis categóricas foram apresentadas em frequências e percentuais e as variáveis numéricas em mediana e desvio-quartílico). O teste de KolmogorovSmirnov foi realizado para avaliar a normalidade e o teste F para avaliar as homogeneidades das variâncias. Para comparação dos dois períodos (antes e depois) foi utilizado, para variáveis categóricas, os testes de Qui-Quadrado, Exato de Fisher e Teste G e para variáveis numéricas o teste de Mann-Whitney. Em todo o trabalho foi adotado o nível de significância de 5\%.

O estudo foi submetido ao Comitê de Ética em Pesquisa (CEP) do Hospital de Clínica Gaspar Vianna, obtendo aprovação sob CAAE $n^{\circ}$ 69517617.4.0000.0016. A pesquisa somente foi iniciada após aprovação pelo CEP, e seguiu os preceitos éticos presentes na Resolução nº666/2012 (Brasil, 2012).

\section{Resultados}

Foram estudados um total de 257 pacientes, sendo 128 do grupo antes do protocolo (grupo AP) e 129 pertencentes ao grupo depois do protocolo (grupo DP), com proporções semelhantes em relação ao sexo. Em relação à média de idade e desvio padrão, no grupo AP foi de $73.5 \pm 23.3$ e no grupo DP $76.0 \pm 17.0$. O tipo de internação em ambos o grupo foi predominantemente clínico, quanto ao desfecho houve aumento no número de altas e redução de transferência e óbitos quando comparados o grupo DP com o grupo AP com significância estatística $(p=0,04)$. Ao avaliar os critérios para classificação quanto a choque séptico e sepse, o grupo DP apresentou aumento de diagnóstico de sepse e redução de diagnóstico de choque séptico quando comparado com o grupo AP, apresentando também significância estatística $(p=0,05)$. A Tabela 1 traz os dados detalhados quanto à caracterização clínica dos pacientes analisados. 
Tabela 1: Distribuição dos pacientes segundo sexo, local de atendimento, tipo de internação, desfecho hospitalar e critérios, atendidos no Hospital Adventista de Belém - Mar/2015 a Dez/2019.

\begin{tabular}{|c|c|c|c|c|c|c|}
\hline \multicolumn{2}{|c|}{ Variáveis } & \multicolumn{2}{|c|}{ Grupo AP } & \multicolumn{2}{|c|}{ Grupo DP } & \multirow{2}{*}{ Valor de $P$} \\
\hline & & $\mathrm{n}=128$ & $\%$ & $\mathrm{n}=129$ & $\%$ & \\
\hline \multirow{2}{*}{ 帝 } & Masculino & 64 & 50.0 & 65 & 50.4 & \multirow{2}{*}{$0.9501^{\mathrm{a}}$} \\
\hline & Feminino & 64 & 50.0 & 64 & 49.6 & \\
\hline 苞 & $\begin{array}{c}\text { Mediana } \pm \\
\text { IIQ }\end{array}$ & \multicolumn{2}{|c|}{$73.5 \pm 23.3$} & \multicolumn{2}{|c|}{$76.0 \pm 17.0$} & $0.4050^{c}$ \\
\hline \multirow{2}{*}{ 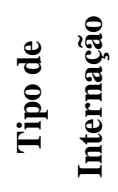 } & Clínica & 123 & 96.1 & 123 & 95.3 & \multirow{2}{*}{$0.9895^{\mathrm{a}}$} \\
\hline & Cirúrgica & 5 & 3.9 & 6 & 4.7 & \\
\hline \multirow{3}{*}{ 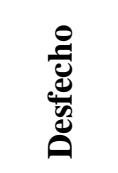 } & Alta & 89 & 69.5 & 104 & 80.7 & \multirow{3}{*}{$0,04^{\mathrm{b}}$} \\
\hline & Transferência & 1 & 0.8 & 0 & 0.0 & \\
\hline & Óbito & 38 & 29.7 & 25 & 19.3 & \\
\hline \multirow{2}{*}{ } & Sepse & 104 & 81.9 & 115 & 89.1 & \multirow[b]{2}{*}{$0,05^{\mathrm{a}}$} \\
\hline & $\begin{array}{l}\text { Choque } \\
\text { Séptico }\end{array}$ & 24 & 18.1 & 14 & 10.9 & \\
\hline
\end{tabular}

AP: Antes do Protocolo; DP: Depois do Protocolo.

a Teste do Qui-quadrado

b Teste Exato de Fisher

${ }^{c}$ Teste de Mann-Whitney

Fonte: Protocolo do Hospital Adventista de Belém.

Todos os pacientes que desenvolveram sepse foram diagnosticados no serviço de emergências da instituição. Na maioria da população estudada, em ambos os grupos o motivo de internação foi clínico $(p=0.9895)$, o tipo de infecção foi comunitário ( $p=0.0019$ ) e o foco pulmonar. Na avaliação do SOFA (Sequential Organ Failure), os pacientes do grupo antes do protocolo apresentaram maior pontuação que os pacientes do grupo depois do protocolo ( $p=0.0003)$.

Após a implantação do protocolo houve redução do tempo de disfunção orgânica, taxa de mortalidade, permanência na UTI e permanência no hospital, ambas com diferença estatística de $\mathrm{p}=0,0015, \mathrm{p}=0,04, \mathrm{p}=0,012 \mathrm{e} \mathrm{p}=0,0401$, respectivamente (Tabela 2 ). 
Tabela 2: Principais características e desfecho - Hospital Adventista de Belém - Mar/2015 a Dez/2019.

\begin{tabular}{lccc}
\hline \multicolumn{1}{c}{ Característica e Desfecho } & Grupo AP (n=128) & Grupo DP (n=129) & Valor de p \\
\hline Tempo de disfunção orgânica (horas) & $25,59 \pm 108,01$ & $0.15 \pm 0.36$ & $0,0015^{\mathrm{c}}$ \\
Taxa de Mortalidade & 29.7 & 19.3 & $0,04^{\mathrm{b}}$ \\
Permanência na UTI (dias) & $19,63 \pm 90,43$ & $1,52 \pm 5,57$ & $0,012^{\mathrm{c}}$ \\
Permanência no Hospital (dias) & $19,32 \pm 28,63$ & $10.28 \pm 11.72$ & $0,0401^{\mathrm{c}}$ \\
\hline
\end{tabular}

b Teste Exato de Fisher

${ }^{c}$ Teste de Mann-Whitney

Fonte: Protocolo do Hospital Adventista de Belém.

$\mathrm{Na}$ avaliação dos custos hospitalares, foi identificada redução na média gasta com cada paciente tanto no custo total da hospitalização quanto no custo total referente apenas ao tempo de permanência na UTI (Tabela 3).

Tabela 3: Custos hospitalares dos pacientes atendidos no Hospital Adventista de Belém - Mar/2015 a Dez/2019.

\begin{tabular}{lcc}
\hline \multicolumn{1}{c}{ Custos Hospitalares } & Grupo AP & Grupo DP \\
\hline *Gasto Total de Hospitalização & $79.510,80 \pm 147745,48$ & $18.951,06 \pm 37052,90$ \\
*Custo Total de UTI & $37.373,38 \pm 62604,65$ & $7.171,89 \pm 19033,51$ \\
\hline
\end{tabular}

Valores expressos em reais. Os resultados são expressos como média \pm desvio padrão.

$* p<0,05$.

Fonte: Protocolo do Hospital Adventista de Belém.

Em relação ao atendimento no Pronto Socorro, observou-se diferença estatisticamente significativa no custo total ( $\mathrm{p}<0,001)$ entre os dois períodos (antes e depois) (Gráfico 1).

Gráfico 1: Custo dos atendimentos no Pronto Socorro Antes e Depois da Implantação do protocolo de sepse.

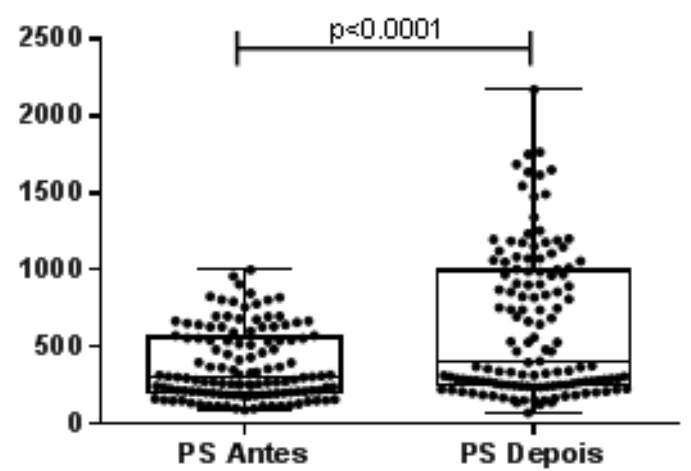

Fonte: Protocolo do Hospital Adventista de Belém.

Em contrapartida em relação ao atendimento na internação, não foi observado diferença significativa no custo total ( $\mathrm{p}=0,0694)$ entre os dois períodos (antes e depois) (Gráfico 2). 
Gráfico 2: Custo dos atendimentos na Internação Antes e Depois da Implantação do protocolo de sepse.

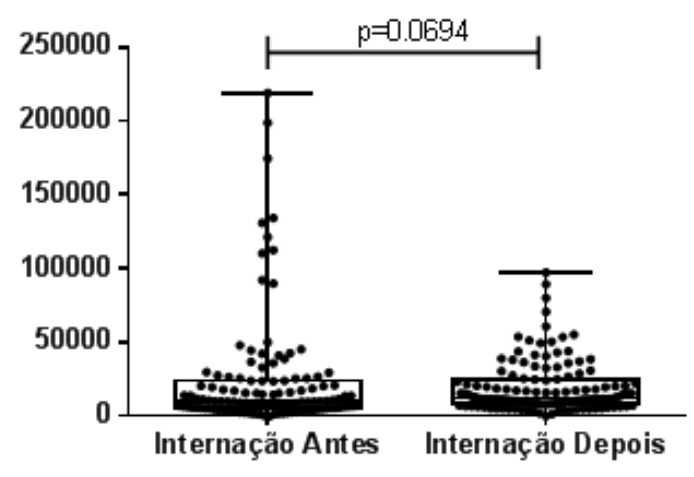

Fonte: Protocolo do Hospital Adventista de Belém.

\section{Discussão}

\subsection{Custos relacionados a assistência}

A sepse se configura como um problema de saúde pública que acarreta grandes gastos aos sistemas de saúde públicos e privados, pois representa uma das 5 principais causas de oneração em hospitais. Esta patologia causa elevada morbimortalidade nos pacientes e necessita de maior tempo de internação, isto corrobora para o cenário de altos custos financeiros à medida que o paciente com sepse possui seis vezes mais custos quando comparado a um paciente não séptico (Sogayar et al., 2008; Chalupka \& Talmor, 2012).

Quando se tratam dos valores totais anuais gastos nos Estado Unidos da América, estes correspondem a R $\$ 17,3$ bilhões de dólares, porém quando considerados os gastos sociais que a sepse trás, esse valor representa apenas $30 \%$ do total gasto. Isso demonstra que os custos vão além da assistência no ambiente hospitalar (Koenig et al., 2010; Silva et al., 2019).

No Brasil os custos da assistência ao cliente com sepse representam em torno de 9,6 mil dólares por paciente. Quando se compara as regiões brasileiras, segundo um estudo realizado por Silva et al. (2019), a região Norte é a que menos gasta com sepse no Brasil, algo em torno de R\$292,54 reais por dia com pacientes com sepse. Por outro lado, a região vizinha, Nordeste, mostrou-se a região que mais gasta diariamente com sepse, $\mathrm{R} \$ 320,70$ reais/paciente/dia (Silva et al., 2019).

Ainda segundo Silva et al. (2019) os gastos demandados pela sepse correspondem de $24 \%$ a $32 \%$ do total de gastos em Unidades de Terapia Intensiva (UTI). Estes valores são atribuídos à necessidade, na maioria dos casos, de reinternação dos pacientes que sofreram de sepse ou choque séptico.

Além disto, o diagnóstico, tratamento, procedimentos mais complexos e os processos atribuídos ao período de internação explicam o alto custo dessa patologia, levando-se em consideração também os custos indiretos associados aos recursos humanos (Silva et al., 2019; Jost et al., 2019).

Vale ressaltar que as disfunções orgânicas causadas pela sepse demandam de terapias substitutivas que elevam o custo do tratamento, além disso, o fato de medicamentos caros e de uma assistência contínua por meio de uma equipe multidisciplinar ampliam as despesas. Notoriamente percebido no gráfico 1, em que se visualiza o custo mais elevado após a implementação do protocolo de sepse na emergência, tendo em vista a necessidade de rastreio e detecção precoce da sepse, com exames laboratoriais, inicio de antibióticos e equipe auditoria do protocolo.

O cálculo do valor gasto no manejo da sepse leva em consideração a terapêutica, diagnóstico e recursos humanos e físicos aplicados a esse paciente conforme o protocolo adotado pela instituição. Esses protocolos são criteriosos e contam com exames, medicamentos e condutas profissionais. Entretanto vale ressaltar a dificuldade em calcular o custo de condutas da equipe de enfermagem, médica e multiprofissional (Silva et al., 2019). Pode-se inferir que não houve diferença estatística pois 
com a detecção precoce eleva-se o custo de diagnóstico e o paciente é tratado imediatamente, podendo aumentar o valor do diagnóstico e do tratamento, em contrapartida há diminuição do tempo de internação, logo o custo de internação não elevou-se significativamente.

Em um estudo realizado em dois hospitais em Joinville/SC foi evidenciada a associação entre custo elevado e paciente mais grave, visto que, os pacientes mais graves e com desfecho em óbito correspondiam a um custo mais elevado quando comparados aos pacientes sobreviventes à sepse. Esse fato pode estar associado a aplicação de mais esforços e maior tempo de internação aos pacientes sépticos graves (Koenig et al., 2010).

O custo da sepse por paciente decai com a idade, ou seja, quanto menor a idade, mais caro se torna o manejo do paciente com sepse. Entretanto vale ressalta que a maior parcela de pacientes com sepse então compreendido na faixa igual ou superior a 65 anos, logo o tratamento do idoso com sepse torna-se cara pela sua grande parcela epidemiológica nesta patologia. O custo de um paciente com menos de 18 anos é de aproximadamente 5.662,90 dólares em detrimento de paciente com mais de 80 anos que correspondem a custos menores por volta de 4.574,00 dólares (Chalupka \& Talmor, 2012).

Um estudo realizado por Taniguchi et al. (2019) em 1.690 UTI traz números relevantes no que diz respeito à disponibilidade de maior recurso para diagnóstico e terapêutica da sepse influindo em um desfecho positivo do paciente. A falta ou pouca disponibilidade de recursos voltados para a sepse influi na possibilidade de sobrevivência ou mortalidade como desfecho do paciente, visto que em países de alta renda a número das taxas de mortalidade vem diminuindo quando comparadas àqueles de baixa renda (Schultz et al., 2017). Contraditoriamente, dados encontrados em nosso estudo apontam que após a aplicação do protocolo de sepse houve melhora no número de altas e redução de óbitos (ainda que não tenha apresentado significância estatística) com redução significativa dos gastos $(p<0,0001)$, indicando que somente os recursos financeiros não são os únicos que impactam na morbimortalidade.

Entretanto há discordância entre autores acerca do assunto disponibilidade de recursos e desfecho positivo, visto que Chalupka e Talmor (2012) mostram a variação entre recursos e mortalidade ao trazer dados em que 10\% dos 309 hospitais pesquisados apresentaram elevação na taxa de mortalidade mesmo superando os custos esperados para o tratamento da sepse, colocando e xeque que não são necessários somente recursos financeiros, mas também uma sistematização do diagnóstico e tratamento, fato que foi adotado na instituição em que nosso estudo foi realizado e demonstrou melhora nos desfechos dos pacientes.

Em pesquisas realizadas em UTI's do Reino Unido, os medicamentos aplicados na terapêutica medicamentosa e de fluidos e os cuidados prestados pela equipe de enfermagem representaram as duas maiores parcelas de despesas com sepse. $\mathrm{O}$ custo do pessoal de enfermagem foi de 317 dólares, o qual corresponde a 53\% do total dos custos diários da UTI, enquanto que os gastos com medicamentos e fluidos foram de 19\% (116,00 dólares) do custo diário total nesse setor (Chalupka \& Talmor, 2012).

No que diz respeito ao agente causador de sepse, este pode alterar o custo para o hospital. As infecções de corrente sanguínea causadas por Candida spp são o dobro mais caras quando comparadas aos custos de bacteremias, esses valores podem ser de 30.219 dólares e 12.305-21.678 dólares para candida e bactérias, respectivamente. A diferença de custos entre as variadas cepas de bactérias também é visualizada à medida que o tratamento de casos de sepse causados por bactérias grampositivos é mais caro do que aquelas causadas por gram-negativos (Chalupka \& Talmor, 2012).

\subsection{Impacto de Protocolos na redução de custos}

Uma revolução administrativa vem acontecendo nos hospitais do país, há algum tempo, na qual suas estruturas passaram a se direcionar para a maneira como são prestados seus serviços, sendo observados detalhes antes não considerados 
importantes. Essa mudança de postura decorre de uma visível transformação por parte dos principais agentes financiadores do sistema (Lagioia et al., 2008).

Rutledge (1996) afirma que no ambiente hospitalar os médicos e os administradores vêm demandando cada vez mais de uma maior interação no que se trata de controle de custos e melhoria da qualidade. Também corrobora afirmando que apesar da equipe médica exercer uma função fundamental nos hospitais e de responder por uma grande porcentagem de todos os custos da instituição, ele dificilmente é informado ou consultado sobre as tomadas de decisões realizadas pelos administradores e o planejamento financeiro. Ainda em suas pesquisas observou-se que o envolvimento do corpo médico na tomada de decisões administrativas promoveu uma diminuição dos custos e uma melhora de desempenho nas atividades desenvolvidas no hospital no geral.

A utilização de protocolos surgiu da engenharia civil, buscando o máximo de eficiência na utilização dos recursos através da criação de modelos de procedimentos a serem utilizados nas construções. Esse raciocínio foi levado e adaptado para a medicina, com a criação de padrões de tratamento para serem utilizados com os pacientes. Na década de 70, foram criados os primeiros protocolos em unidades intensivas, objetivando padronizar o tratamento e se certificar de que nada seria omitido no diagnóstico do paciente. Porém, somente no final da década de 1980 os protocolos médicos foram implementados de fato (Nogueira, 2003).

Lagioia et al. (2008), afirma que o uso dos protocolos auxilia a equipe médica na decisão da melhor intervenção a ser usada conforme a complexidade da patologia encontrada. Dessa forma, os protocolos podem auxiliar não apenas na alteração dos custos, na média de permanência e o nível de mortalidade consequente de possíveis intervenções médicas, mas pode também verificar a morbidade decorrente de mudanças nos sintomas e sinais fisiológicos dos pacientes. Tendo como objetivo determinar as melhores práticas durante o tratamento e identificar as informações críticas que sirvam de suporte clínico para a tomada de decisões.

Além da equipe médica, Lima et al. (2015), em sua pesquisa sobre um protocolo de prevenção de úlceras de pressão, apontam que os enfermeiros, na condição de coordenadores de planos de cuidados dos pacientes e gerentes das unidades assistenciais, necessitam conhecer e se utilizar de informações financeiras voltadas a novas propostas de trabalho e novas tecnologias.

Segundo o Instituto Latino Americano de Sepse (2018), a sepse é uma síndrome prevalente, com altas taxas de morbidade e mortalidade além de altos custos. Seu reconhecimento precoce e tratamento adequado são fatores primordiais para a mudança deste cenário. A efetivação de protocolos clínicos gerenciados é uma ferramenta útil neste contexto, visando auxiliar as instituições na padronização do atendimento ao paciente séptico, diminuindo desfechos negativos e proporcionando melhor efetividade do tratamento. Tais informações corroboram com o achado nesse estudo, no qual percebe-se melhora da efetividade do tratamento ao analisar a taxa de óbitos e altas após a implantação do protocolo.

De acordo com o ponto de vista econômico, a sepse representa grande custo para o sistema de saúde, assim como importante diminuição de produtividade vinda de longas permanências hospitalares. A implantação de protocolos para detecção e tratamento precoce estão voltados para a diminuição de taxas de morbidade, mortalidade e além dos custos associados à sepse (Koenig et al., 2010). De modo semelhante, observamos a redução do custo total de gastos no período anterior e posterior a implantação do protocolo de sepse, com diferença estatisticamente significativa $(p<0,001)$, demonstrando a melhoria que os protocolos representam no que tange a economia dos serviços de saúde.

Estimativas apontam que o dispêndio direto na sepse nos Estados Unidos da América anualmente é de cerca de 17 bilhões de dólares, representando 30\% do custo total da doença, sendo também considerados os custos sociais (Sogayar \& Silva et al., 2005; Koenig et al., 2010). Um estudo multicêntrico e prospectivo analisou os custos diretos do tratamento de sepse e, hospitais do Brasil que demonstraram menos recursos direcionados para os sobreviventes do que para os não 
sobreviventes, estes apresentaram estadia mais longas na Unidade de Terapia Intensiva, desta forma foram submetidos a um maior número de procedimentos diagnósticos e terapêuticos durante a internação (Sogayar et al., 2008).

Condizente com o relatório da Campanha Sobrevivendo à Sepse, em 2003 foram notificados 398.000 casos e 227.000 mortes por choque séptico no Brasil. Aproximadamente R \$ 17,3 bilhões por ano são usados para o tratamento de pacientes sépticos, sendo que R $\$ 10$ bilhões deste são direcionados a pacientes que evoluem a óbito, criando um impacto econômico. As elevadas taxas de mortalidade do choque séptico e sepse grave, bem como os elevados custos associados ao seu tratamento tornam evidente a necessidade de sua profilaxia e diagnóstico precoce (Instituto Latino Americano de Sepse, 2018). Schorr, Micek, Jackson \& Kollef (2007) encontraram uma redução significativa nos custos diretos do tratamento de pacientes com sepse grave logo após a implementação de protocolos de tratamento recomendados no Surviving Sepsis Campaign.

Os estudos de Ramão et al. (2018) notaram uma melhora significativa no fluxo de atendimento no pronto socorro, após a implantação do protocolo de trombólise em acidente vascular encefálico isquêmico, otimizando o tempo entre a admissão, o diagnóstico e o tratamento dos pacientes acometidos pela condição clínica citada. Assim, obteve-se melhora no prognóstico clínico dos casos observados/tratados. Houve uma diminuição no tempo de internação e, consequentemente, redução dos custos e dos índices de infecção hospitalar, oferecendo melhor giro dos leitos do hospital, melhorando o fluxo e a admissão dos pacientes politraumatizados em enfermarias ou UTI.

O uso de protocolos auxilia na melhoria da assistência não somente em casos de sepse, mas em diversas situações, como explicitado no estudo de Souza \& Lima Júnior (2014), no qual demonstram que a criação de protocolos para cirurgia com alta no mesmo dia se mostra uma ferramenta importante na gestão hospitalar, ajudando a diminuir custos, aumentando a produtividade e reduzindo o tempo de espera pelos procedimentos.

Identificou-se no presente estudo a redução tanto da mortalidade, quando dos custo, exemplificando a excelente utilização dos protocolos na prática clínica. Em consonância com esses resultados, estudos demonstram a efetividade da implementação de protocolos assistenciais gerenciados, baseados nas diretrizes, impactando positivamente na evolução dos pacientes. No Brasil, foi publicada, em parceria com o ILAS, a casuística de uma rede de hospitais. Utilizando a estratégia de implementação do instituto, obteve-se diminuição importante da letalidade ao longo dos trimestres do processo (de 55\% para 26\%). Uma cuidadosa análise farmacoeconômica mostrou que o processo, além de efetivo, economizava custos, em termos de anos de vida salva com qualidade (Noritomi et al., 2014).

\section{Considerações Finais}

É perceptível que os custos com sepse configuram um problema de saúde pública tanto no âmbito nacional, quanto internacional. Contudo tais gastos podem ser reduzidos com a implantação de protocolos que visem padronizar a assistência prestada aos pacientes e com isso influenciar positivamente na redução do tempo de internação, custos com a internação e mortalidade.

A realização do presente estudo traz informações úteis para que protocolos semelhantes sejam aplicados em demais hospitais em que não haja padronização do tratamento de sepse. Ademais, o estudo demonstra que a taxa de resolução dos casos de sepse não estão atrelados ao maior custo do tratamento, mas também é influenciado pela organização e sistematização dos procedimentos desde o diagnóstico ao seguimento do paciente pós alta.

Por avaliar apenas um serviço de assistência a pacientes com sepse, esse estudo apresenta como limitação o fato de ter resultados somente de uma população específica, sendo necessária a realização de outros estudos em regiões diferentes da aqui analisada.

Frente a escassez de estudo que abordem o custo benefício da utilização de protocolos de sepse na região norte do país, é esperado novos estudos sejam realizados a fim de identificar relatar a experiência de outros serviços com a implantação 
de protocolo de sepse. Adicionalmente, este estudo servirá como subsidio para futuras pesquisas que abordem essa temática, além de servir como possível fonte para adequação dos protocolos em outras instituições frente ao custo benefício aqui demonstrado.

\section{Agradecimentos}

Agradecemos a equipe de pesquisadores e aos profissionais de saúde da assistência do Hospital Adventista de Belém envolvidos no planeamento e execução do presente estudo.

\section{Referências}

Brasil. (2012). Conselho Nacional de Saúde. Resolução 466/ de 12 de dezembro de 2012: Trata de pesquisas em seres humanos e atualiza a resolução 196. Diário Oficial da União. http://conselho.saude.gov.br/resolucoes/2012/Reso466.pdf.

Brasil. Ministério da Saúde. (2014). Documento de referência para o Programa Nacional de Segurança do Paciente. Ministério da Saúde; Fundação Oswaldo Cruz; Agência Nacional de Vigilância Sanitária. Ministério da Saúde. https://bvsms.saude.gov.br/bvs/publicacoes/documento_ referencia_programa_nacional_seguranca.pdf.

Bruhn A. C., Pairumani R. M. \& Hernandez G. P. (2011). Manejo del paciente, em shock séptico. Revista Médica Clínica Las Condes, 22(3):293-301. https://doi.org/10.1016/S0716-8640(11)70429-1.

Chalupka, A. N. \& Talmor, D. (2012). The Economics of Sepsis. Critical Care Clinics, 28(1), 57-76. https://doi.org/10.1016/j.ccc.2011.09.003.

Dellinger R. P., Levy M. M., Rhodes A., Annane D., Gerlach H., Opal S. M., et al. (2013). Surviving sepsis campaign: international guidelines for management of severe sepsis and septic shock, 2012. Critical Care Medicine and Intensive Care Medicine, 41:580-637. https://doi.org/10.1007/s00134-0122769-8.

Espichán M. M. (2010). Strategies for improving survival in patients with severe sepsis. Acta Médica Peruana, 27(4):302-309. https://amp.cmp.org.pe/index.php/AMP/article/view/1421.

Hernández G., Bruhn A., Castro R. \& Reguera T. (2012) The holistic view on perfusion monitoring in septic shock. Critical Care, 18:280-6. https://doi.org/10.1097/MCC.0b013e3283532c08.

Ilas Online. (2015). Instituto Latino Americano da Sepse. http://www.ilasonline.org.br/index-modal.php\#close.

Instituto Latino Americano de Sepse. (2018). Implementação De Protocolo Gerenciado De Sepse Protocolo Clínico - Atendimento Ao Paciente Adulto Com Sepse / Choque Séptico. Instituto Latino Americano de Sepse. https://ilas.org.br/assets/arquivos/ferramentas/protocolo-de-tratamento.pdf.

Instituto Latino-Americano para Estudos da Sepse (ILAES). (2015). Sepse: um problema de saúde pública. Instituto Latino-Americano para Estudos da Sepse, CFM. https://ilas.org.br/assets/arquivos/upload/Livro-ILAS(Sepse-CFM-ILAS).pdf.

Jost, M. T., Machado, K. P. M., De Oliveira, A. P. A., da Costa Linch, G. F., Paz, A. A., Caregnato, R. C. A. \& Blatt, C. R. (2019). Morbimortalidade e custo por internação dos pacientes com sepse no Brasil, Rio Grande do Sul e Porto Alegre. Journal of Epidemiology and Infection Control, 9(2). https://doi.org/10.17058/reci.v9i2.12723.

Koenig, A., Picon, P. D., Feijó, J., et al. (2010). Estimate of the economic impact of implementing an in hospital protocol for the early detection and treatment of severe sepsis in public and private hospitals in southern Brazil. Rev Bras Ter Intensiva. https://doi.org/10.1590/S0103-507X2010000300001.

Lagioia, U. C. T., Ribeiro Filho, J. F., Falk, J. Á., Libonati, J. J. \& Lopes, J. E. G. (2008). A gestão por processo gera melhoria de qualidade e redução de custos: o caso da unidade de ortopedia e traumatologia do Hospital das Clinicas da Universidade Federal de Pernambuco. Rev Contab Financ. 19(48), 77-90. https://doi.org/10.1590/S1519-70772008000300007.

Lima, A. F. C., Castilho, V., Rogenski, N. M. B., Baptista, C. M. C. \& Rogenski, K. E. (2015). Custos da implantação de um protocolo de prevenção de úlceras por pressão em um hospital universitário. Rev. Eletr. Enf. 17(4). http://dx.doi.org/10.5216/ree.v17i4.31051.

Nogueira, L. C. L. (2003). Gerenciando pela qualidade total na saúde. Editora de Desenvolvimento Gerencial.

Noritomi, D. T., Ranzani, O. T., Monteiro, M. B., Ferreira, E. M., Santos, S. R., Leibel F. \& Machado, F. R. (2014). Implementation of a multifaceted sepsis education program in an emerging country setting: clinical outcome sand cost-effectiveness in a long-term follow-up study. Intensive Care Med., 40(2):18291. https://doi.org/10.1007/s00134-013-3131-5.

Pereira, A. S., Shitsuka, D. M., Parreira, F. J., \& Shitsuka, R. (2018). Metodologia da pesquisa científica. UFSM. https://repositorio.ufsm.br/bitstream/h andle/1/15 824/Lic_Computacao_Metodologia-Pesquisa-Cientifica.pdf?sequence=1.

Pérez, A. L., Rosell. E. C., Lacosta, M. D., Dardet, C. A., Selles, J. U. \& Mendonza, C. L. M. (2012). Clinical pathway intervention compliance and effectiveness when used in the treatment of patients with severe sepsis and septic shock at an Intensive Care Unit in Spain. Revista Latino-Americana de Enfermagem, 20(4):09. https://doi.org/10.1590/S0104-11692012000400002. 
Research, Society and Development, v. 10, n. 5, e23110514931, 2021

(CC BY 4.0) | ISSN 2525-3409 | DOI: http://dx.doi.org/10.33448/rsd-v10i5.14931

Ramão, G. B., Ferraz, R. R. N. \& Guirado, G. M. P. (2018). Redução dos custos e do tempo de internação em um hospital público da capital paulista com a implementação do protocolo de trombólise em acidente vascular cerebral isquêmico. Revista de Tecnologia Aplicada, 7(1) 3-10. http://dx.doi.org/10.21714/2237-3713rta2018v7n1p03.

Rocha, L. L., Pessoa, C. M. S., Corrêa, T. D., Pereira, A. J., Assunção, M. S. C. \& Silva, E. (2015). Current concepts on hemodynamic support and therapy in septic shock. Revista Brasileira de Anestesiologia, 65(5):395-402. https://doi.org/10.1016/j.bjane.2014.11.006.

Romero C. \& Hernández G. (2013). Initial resuscitation bundle and monitoring tissue perfusión in severe sepsis. Revista Medicina Chile, 141:1173-1181. https://doi.org/10.4067/S0034-98872013000900010.

Rutledge, V. R. (1996). Hospital physicianalignment: a model for success. Oncology Issues. Associationof Community Centers. 11(6), 18-20. https://doi.org/10.1080/10463356.1996.11904645.

Schorr, A. F., Micek, S. T., Jackson, W. L. J. \& Kollef, M. H. (2007). Economic implications of an evidence-based sepsis protocol: can we improve outcomes at lower costs? Crit Care Med, 35(5):1257-62. https://doi.org/10.1097/01.CCM.0000261886.65063.CC.

Schultz, M. J. et al. (2017). Current challenges in the management of sepsis in ICUs in resource-poor settings and suggestions for the future. Intensive Care Medicine, 43(5), 612-624. https://doi.org/10.1007/s00134-017-4750-z.

Silva, L. M. N., Carneiro, R. F., Oliveira, L. P. L., Raposo, L. M., Oliveira, T. A. \& Sugita, D. M. (2019). Levantamento do custo da internação por septicemia com base em protocolo atual de manejo da doença. Revista Educação em Saúde, 7(1), 47-57. https://doi.org/10.29237/2358-9868.2019v7i1.p47-57.

Sogayar, A. M., Silva, E., Cal, R. G., Beer, I., Akamine, N., Safi, J. J. \& Kayath, M., the Costs Group. (2005). What are the direct costs of sepsis treatment in Brazilian ICUs? Crit Care, 9(2):P112. https://doi.org/10.1186/cc3656.

Sogayar, A. M., Machado, F. R., Rea-Neto, A., Dornas, A., Grion, C. M., Lobo, S. M., Tura, B. R., Silva, C. L., Cal, R. G., Beer, I., Michels, V., Safi, J., Kayath, M. \& Silva E. (2008). Costs Study Group - Latin American Sepsis Institute. A multicentere, prospective study to evaluate costs of septic patients in Brazilian intensive care units. Pharmacoeconomics, 26(5):425-34. https://doi.org/10.2165/00019053-200826050-00006.

Souza, M. E. G. \& Lima Junior, Z. B. (2014). Impacto do protocolo de day surgery para colecistectomia videolaparoscópica no Hospital Universitário Lauro Wanderley. Biblioteca Setorial do Centro de Ciências Médicas.

Taniguchi, L. U., Azevedo, L. C. P., Bozza, F. A., Cavalcanti, A. B., Fernanda, E. M. F., Carrara, S. A., Sousa, J. L., Salomão, R. \& Machado, F. R. (2019). Disponibilidade de recursos para tratamento da sepse no Brasil: uma amostra aleatória de instituições brasileiras. Rev Bras Ter Intensiva, 31(2), 193-201. https://doi.org/10.5935/0103-507x.20190033. 